\title{
Circuit QED Bright Source for Chiral Entangled Light Based on Dissipation
}

\author{
Fernando Quijandría, ${ }^{1}$ Diego Porras, ${ }^{2}$ Juan José García-Ripoll, ${ }^{3}$ and David Zueco ${ }^{1,4}$ \\ ${ }^{1}$ Instituto de Ciencia de Materiales de Aragón y Departamento de Física de la Materia Condensada, \\ CSIC-Universidad de Zaragoza, Zaragoza E-50012, Spain \\ ${ }^{2}$ Departamento de Física Teórica I, Universidad Complutense, Madrid E-28040, Spain \\ ${ }^{3}$ Instituto de Física Fundamental, IFF-CSIC, Serrano 113-bis, Madrid E-28006, Spain \\ ${ }^{4}$ Fundación ARAID, Paseo María Agustín 36, Zaragoza 50004, Spain \\ (Received 29 December 2012; revised manuscript received 1 April 2013; published 15 August 2013)
}

\begin{abstract}
We present a scalable and tunable framework for the quantum simulation of critical dissipative models based on a circuit QED cavity array interacting with driven superconducting qubits. We will show that the strongly correlated many-body state of the cavities can be mapped into the state of propagating photons in a transmission line. This allows not only for an efficient way of accessing the correlations in the manybody system, but also provides a bright source of chiral entangled light where directionality and entanglement are assisted by collective phenomena and breaking of reflection symmetry.
\end{abstract}

DOI: 10.1103/PhysRevLett.111.073602

PACS numbers: 42.50.Dv, 03.67.Bg, 85.25.Cp

The controlled generation of quantum states of light is crucial for applications both in quantum information processing [1] and precision measurements [2]. While Gaussian entangled states of light can be generated through nonlinear optical interactions [3], the field of superconducting quantum circuits and circuit QED provides us with more efficient tools to explore such applications [4]. In this respect, we recall the preparation of single-mode squeezing [5], two-mode squeezing [6,7], broadband squeezed light [8], single-mode nonclassical states of light [9], and entanglement [10], as well as the extremely precise tomography both in cavities [9] and in open transmission lines [11].

Two distinctive features of circuit QED (CQED) are the precise positioning of quantum emitters and their local control through driving fields or, more recently, dissipation [12]. These ingredients enable a paradigm shift in the control of light-matter interaction. First, a controlled separation between quantum emitters allows us to tailor collective effects like directionality and interference, with an accuracy that can hardly be matched by atomic systems. Second, these collective effects can now be embodied with dissipative dynamics [13], introducing novel dissipative quantum many-body phenomena [14-16], such as nonequilibrium phase transitions and dissipative criticality classes.

In this Letter, we explore the generation of strongly correlated states of microwave light using dissipative phenomena in arrays of coupled cavities. We pursue the generation of many-body entanglement by means of a scalable framework of tunable squeezed dissipators. Inspired by laser cooling, we introduce a simple design based on driven qubits that allows the quantum simulation of a wide variety of Liouvillians exhibiting criticality [16]. Most importantly, we demonstrate that the quantum state of these cavities can be efficiently mapped to the state of propagating photons. This not only gives access to the correlation and entanglement of the many-body system but, as the title of this Letter suggests, gives rise to a distinct source of entangled light.

The device that we present is a quantum metamaterial [17] that embeds a regular array of dissipative quantum emitters, merging a variety of physical effects into a single CQED device: (a) nonperturbative sideband transitions in the qubit-cavity couplings, engineered by suitable qubit drivings; (b) an incoherent qubit repumping mechanism based on a combination of local reservoirs with the qubit driving; (c) directional emission of light in the cavity array supported both by a collective interference and the band engineering in this metamaterial. Through a detailed theoretical study of this setup, we prove that our device has the following properties. (i) It acts as a source of entangled light, created by a dissipative process that arises from a combination of precisely tuned drivings with fast qubit decays. (ii) The degrees of entanglement and squeezing, as well as the frequency of the entangled modes, can be tuned by means of the periodic drivings. (iii) It implements a chiral dissipative process with broken reflection symmetry, induced by the phase pattern of the driving. In practice, this effect allows us to control the direction and momentum of the emitted entangled modes. (iv) The setup can be used to study dissipative many-body collective effects, both because of its nontrivial collective steady state, but also because the system undergoes a dissipative quantum phase transition for suitable parameters.

The setup.-Our dissipative source of chiral quantum light is schematically drawn in Fig. 1(a). It consists of a 1D arrangement of coupled cavity-qubit units where the qubit gaps are periodically and uniformly driven, and where each unit is coupled to an independent thermal bath to which they can dissipate. We describe the coherent part of the cavity-qubit (CQ) units using the Hamiltonian (throughout this Letter $\hbar=1$ ) 


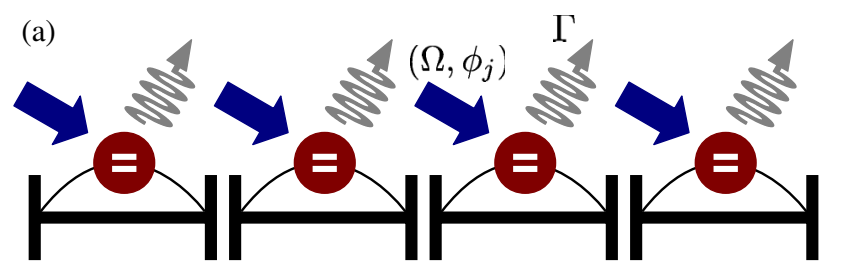

(b)

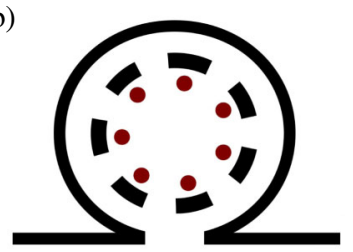

(c)

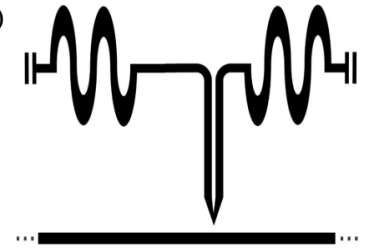

FIG. 1 (color online). (a) Setup of the system studied. Each superconducting resonator is coupled to a qubit. The resonators are coupled to each other, e.g., capacitively. We have also drawn arrows representing the classical driving and the losses. (b) Sketch for the quantum light source. The coupled cavity can be embedded inside an open transmission line. The entanglement generated in the array is mapped onto the line. (c) Detail for the resonator line coupling. We suggest a pointlike coupling and a possible circuit design (see text).

$H_{\mathrm{CQ}}=\sum_{j}\left(\omega_{\mathrm{r}} a_{j}^{\dagger} a_{j}+\frac{\epsilon}{2} \sigma_{j}^{z}+g \sigma_{j}^{x} X_{j}\right)+J \sum_{\langle i, j\rangle} X_{j} X_{i}$,

where $\sigma_{j}^{z}$ labels a qubit state with frequency $\epsilon, a_{j}^{\dagger}$ represents the creation of a photon with frequency $\omega_{r}$, and $X_{j}=$ $\left(a_{j}+a_{j}^{\dagger}\right)$. The Hamiltonian includes both cavity-qubit, $g$, and intercavity coupling, $J$. For moderate couplings and no resonant driving, the cavity-qubit and cavity-cavity interactions can be modeled in the rotating wave approximation (RWA), forming the so called Jaynes-Cummings lattice (JCL), with $\sum_{j} g\left(\sigma_{j}^{+} a_{j}+\right.$ H.c. $)+\sum_{\langle i j\rangle} J\left(a_{i}^{\dagger} a_{j}+\right.$ H.c. $)$. Analogously to the Bose-Hubbard model, it supports an insulator-superfluid quantum phase transition [18-23], which originates from the competition between the repulsive nonlinearity induced by the two-level system and the hopping term $J$. A promising field to implement such many-body systems is CQED, as already, chips with dozens of coupled identical resonators have been reported $[24,25]$.

In contrast with ordinary JCLs, for us the qubits do not act as a source of nonlinearity, but rather mediate an engineered dissipation. Furthermore, our model strongly relies on the use and control of external qubit drivings. All together, the dynamics is given by a Linblad-type master equation [26] that reads

$$
\begin{aligned}
d_{t} \varrho= & -i\left[H_{\mathrm{CQ}}+H_{\mathrm{drive}}, \varrho\right] \\
& +\Gamma \sum_{j}\left(\sigma_{j}^{-} \varrho \sigma_{j}^{+}-\frac{1}{2}\left\{\sigma_{j}^{+} \sigma_{j}^{-}, \varrho\right\}\right) .
\end{aligned}
$$

Throughout this Letter, we neglect radiative losses, which can be straightforwardly included in our formalism and do not modify qualitatively the discussion below. The first important ingredient in this model is a uniform two-tone driving of all qubit gaps

$$
H_{\text {drive }}=\sum_{n=1,2} \sum_{j} \lambda_{n} \cos \left(\Omega_{n} t+\phi_{n, j}\right) \sigma_{j}^{z}
$$

with tunable phases, $\phi_{1,2 j}$. As we will see, this driving plays a major role in the collective effects. The second important ingredient is the cavity and qubit dissipations, $\kappa$ and $\Gamma$. In this Letter, we work in the limit of strong qubit decay, $\Gamma \gg g$, in which the quantum emitter customizes the effective environment of the photons.

Dissipation engineering.-Dissipation is normally regarded as the worst enemy for preserving quantum coherence in general and entanglement in particular. However, an appropriately engineered dissipation is a very efficient and robust way to drive a system to the desired quantum state. This powerful idea has been considered not only on single-particle models such as laser cooling [27,28] and cat state generation [29,30], but also more recently in the engineering of strongly correlated phases [31] and entanglement [32-35].

The principle underlying all these examples is a tailored system-bath interaction, which allows us to engineer the dissipator $\mathcal{L}$ in the master equation governing the irreversible dynamics, $\partial_{t} \rho=\mathcal{L} \rho$. These dissipators drive the system of interest to its stationary state, $\mathcal{L} \varrho^{*}=0$. In most relevant cases, Davies' theory assures convergence to $\varrho^{*}$ for any initial condition [36], thus, avoiding the need to initialize quantum states. All this together makes dissipation engineering a promising new paradigm for quantum information processing [14,15,37].

Our source of entangled light is also based on engineered dissipation. In absence of driving, the setup from Fig. 1 thermalizes via Eq. (2) to a photon vacuum. However, as soon as we modulate the qubit energy levels, the effective dissipation changes its asymptotic state, $\rho^{*}$, to a product of two-mode entangled states. To make our arguments clear, we will first sketch the main ideas using a single qubit-cavity system and then introduce the photon hopping, $J$, leaving all details for the Supplemental Material [38].

Following Refs. [27,28,39], we prove in three steps that a bad qubit can cool a cavity to a squeezed vacuum. First, we adopt an interaction picture with respect to the qubit, resonator and driving, $\tilde{H}_{\mathrm{CQ} 1}=g\left(a e^{-i \omega_{\mathrm{r}} t}+\right.$ H.c. $) \times$ $\left(\sigma^{+} e^{i f(t)}+\right.$ H.c. $)$, where $f(t)=\epsilon t+\sum_{\alpha=1,2}\left(\lambda_{\alpha} / \Omega_{\alpha}\right) \times$ $\sin \left(\Omega_{\alpha} t+\phi_{\alpha}\right)$ includes the periodic driving (3). Next, under the assumption of weak driving, we use the JacobiAnger expansion [40], retaining terms up to order $\left(\lambda_{\alpha} / \Omega_{\alpha}\right)$. The result in the RWA is

$$
\tilde{H}_{\mathrm{CQ} 1}=\bar{g}\left(b^{\dagger} \sigma^{-} e^{-i \Delta t}+\text { H.c. }\right),
$$

for driving frequencies $\Omega_{1,2}=\Delta+\epsilon \pm \omega_{r}$. Note that the qubit is now coupled to the squeezed resonator modes 
$b=a+\eta e^{i q} a^{\dagger}, \quad \eta=\frac{g_{2}}{g_{1}}, \quad q=\phi_{1}-\phi_{2}$.

Here and following, we consider, for simplicity, unnormalized squeezed modes: $\left[b, b^{\dagger}\right] \neq 1$. The squeezing and the effective coupling strength, $\bar{g}=g \times g_{1} e^{-i \phi_{1}}$, are determined by the external driving, $g_{1}=J_{1}\left(2 \lambda_{1} / \Omega_{1}\right) \times$ $J_{0}\left(2 \lambda_{2} / \Omega_{2}\right)$, and $g_{2}=J_{0}\left(2 \lambda_{1} / \Omega_{1}\right) J_{1}\left(2 \lambda_{2} / \Omega_{2}\right)$, through Bessel functions. The final step consists in an adiabatic elimination following the hierarchy of time scales $\epsilon \gg$ $\Gamma \gg \omega_{r}, g, \Delta$. The first inequality validates Eq. (2) since it allows us to treat the qubit dissipation in a weak-coupling or Lindblad-type master equation. The second inequality justifies not only the RWA in the previous steps, but it also indicates that the qubit relaxation, $\Gamma$, is faster than its interaction with the cavity, and thus, it may be adiabatically eliminated. The result of these manipulations is a master equation, $d_{t} \varrho=\left(2 \bar{g}^{2} / \Gamma\right)\left(2 b \varrho b^{\dagger}-\left\{b^{\dagger} b, \varrho\right\}\right)$, that cools the resonator to the vacuum of the squeezed mode, $b$.

What happens when the on-site squeezing of each resonator competes with a coupling between resonators? Since the dynamics of both processes happens on a different operator basis, one would expect a competition between these phenomena and even some phase transition. For answering this problem, we now move on to the coupled Hamiltonian (1). In the simplest case of translational invariance and periodic boundary conditions, we can introduce momentum space modes $a_{k}=N^{-1 / 2} \sum_{j} e^{-i k j} a_{j}$ $(k \in 2 \pi / N \times \mathbb{Z}$ ), and show that the problem is similar to the single resonator case. More precisely, following the same definitions and approximations as before, we obtain the effective master equation in the appropriate interaction picture (see Supplemental Material [38]),

$d_{t} \varrho=\sum_{k}-i \omega_{k}\left[a_{k}^{\dagger} a_{k}, \varrho\right]+\frac{2 \bar{g}^{2}}{\Gamma}\left(2 b_{k} \varrho b_{k}^{\dagger}-\left\{b_{k}^{\dagger} b_{k}, \varrho\right\}\right)$.

The chain of qubits is now cooling the resonators to the vacuum of two-mode-squeezed operators $b_{k}=a_{k}+$ $\eta a_{-k+q}^{\dagger}$ with dispersion relation $\left(J \ll \omega_{r}\right) \quad \omega_{k}=$ $\Delta+2 J \cos (k)$. Let us remark how the external driving fully determines the properties of the asymptotic state. In particular, while the phase of the driving, $q=\phi_{1}-\phi_{2}$, selects the pairing between modes in $b_{k}$, we will show that the choice of frequencies, $\Delta=\left(\Omega_{1}+\Omega_{2}-2 \epsilon\right) / 2$, customizes the band structure and the amount of entanglement. These are the main practical results in this Letter.

Entanglement in the stationary solution.-After the adiabatic elimination, the effective master equation (6) can be written as a direct sum of quadratic dissipators acting on the Fock spaces of the operators $\left(b_{k}, b_{-k+q}\right)$. Consequently, the asymptotic state of the master equation will be a product of Gaussian states in each of these Hilbert spaces, $\varrho^{*}=\otimes \rho_{k,-k+q}^{*}$. Each of the final density matrices $\rho_{k,-k+q}^{*}$ will be fully characterized by the first and second moments of the operators $R^{(k)}=\left\{Q_{k}, P_{k}, Q_{-k+q}, P_{-k+q}\right\}$, with $Q_{k}=1 / \sqrt{2}\left(a_{k}^{\dagger}+a_{k}\right)$ and $P_{k}=-i / \sqrt{2}\left(a_{k}^{\dagger}-a_{k}\right)$. In particular, the first moments are all zero, while the second moments are conveniently grouped in the twomode covariance matrix

$$
\hat{\gamma}_{k}=\frac{1}{2}\left\langle R_{l}^{(k)} R_{m}^{(k)}+R_{m}^{(k)} R_{l}^{(k)}\right\rangle=\left(\begin{array}{cc}
\alpha & \beta_{k} \\
\beta_{k} & \alpha
\end{array}\right) .
$$

This features a diagonal matrix $\alpha=\left(1+\eta^{2}\right) / 2(1-$ $\left.\eta^{2}\right) \rrbracket_{2}$, and two nonzero off-diagonal blocks

$$
\beta_{k}=\frac{\eta}{\mathcal{E}_{k,-k+q}^{2}+\left(1-\eta^{2}\right)^{2}}\left(\begin{array}{cc}
\eta^{2}-1 & \mathcal{E}_{k,-k+q} \\
\mathcal{E}_{k-k+q} & \eta^{2}-1
\end{array}\right) .
$$

Note that the most relevant parameter is

$$
\begin{aligned}
\mathcal{E}_{k,-k+q} & =\Gamma\left(\omega_{k}+\omega_{-k+q}\right) / 4 g_{1}^{2} \\
& =\Gamma[\Delta+2 J \cos (q / 2) \cos (k-q / 2)] / 2 g_{1}^{2},
\end{aligned}
$$

because it determines the degree of entanglement between the pairs of modes, $(k,-k+q)$. We quantify this through the logarithmic negativity (see the Supplemental Material [38]), which is

$$
E_{N}=-\log _{2}\left(\frac{1+\eta^{2}}{1-\eta^{2}}-\frac{2 \eta}{\sqrt{\mathcal{E}_{k,-k+q}^{2}+\left(1-\eta^{2}\right)^{2}}}\right),
$$

whenever $\mathcal{E}_{k,-k+q}<\left(1-\eta^{2}\right)^{3 / 2} / \eta$ and zero elsewhere.

Figure 2 shows a contour plot of $E_{N}$ in terms of both $\mathcal{E}_{k,-k+q}$ and $\eta$, together with some sections for fixed $\mathcal{E}$. Let us remark how entanglement significantly grows when $\mathcal{E}_{k,-k+q}$ approaches 0 and when $\eta$ approaches 1 . A qualitative explanation follows from the master equation (6): when $\mathcal{E}_{k,-k+q}$ approaches zero, it means that $\omega_{k}+\omega_{-k+q}=0$. Consequently, the terms that generate entanglement, $a_{k}^{\dagger} \rho a_{-k+q}^{\dagger}-\left\{a_{k}^{\dagger} a_{-k+q}^{\dagger}, \varrho\right\}$ and $2 a_{k} \varrho a_{-k+q}-\left\{a_{k} a_{-k+q}, \varrho\right\}$, do not oscillate and are not suppressed. Moreover, since the

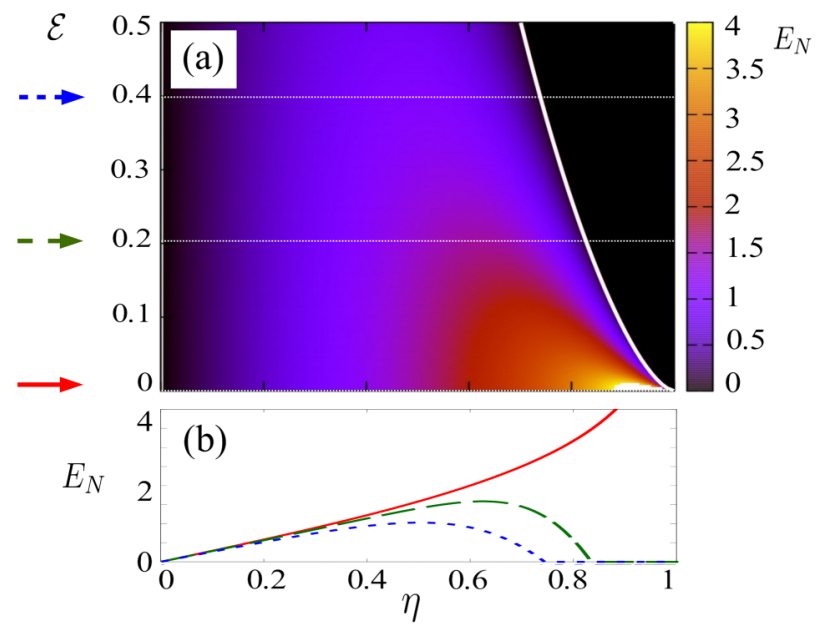

FIG. 2 (color online). (a) Logarithmic negativity $E_{N}$ from Eq. (10). (b) Cuts of (a) at the values of $\mathcal{E}=0,0.2$, and 0.4 (lower), as arrow-marked in (a). For values of $\mathcal{E}$ above $0.5, E_{N}$ becomes negligible. 
strength of those terms grows as $\eta^{2}$ and we know that $\eta \leq 1$ by definition, the optimal amount of entanglement is found when $\eta$ approaches 1 . We focus on the case $\eta \leq 1$, which corresponds to cooling to a squeezed vacuum, since the case $\eta>1$ does not have a well defined steady state, thus, signaling an instability in the system.

We finish by an estimation of the amount of entangled mode pairs in momentum space. Figure 2 shows that what really limits the entanglement is $\mathcal{E}_{k,-k+q}$. The bigger the entanglement, the closer $\mathcal{E}_{k,-k+q}$ must be to 0 . It turns out that $\mathcal{E}_{k,-k+q}=0$ iff $2 J / \Delta>1$ [cf. below Eq. (8)].

Criticality and phase transitions.-We have found that entanglement diverges in the limit $\eta \rightarrow 1$ when $\mathcal{E}_{k,-k+q}=$ 0 . In this limit, it is easy to show that $\rho^{*}$ becomes a product of two-mode Einstein-Podolsky-Rosen state [41], with a diverging correlation length. This is an example of a critical point which is entirely driven by dissipation [16].

However, criticality in dissipative systems is characterized by the vanishing of the $\mathcal{L}$ eigenvalue with the largest real part (closest to zero), $\operatorname{Re}\left(\lambda_{1}\right) \rightarrow 0$ [42]. A practical consequence is that the relaxation time becomes infinite, $T_{1} \sim\left[\operatorname{Re}\left(\lambda_{1}\right)\right]^{-1} \rightarrow \infty$, close to the transition point. In our case, $T_{1} \sim(1-\eta)^{-1}$. Consequently, the rise in the time needed to reach the stationary entangled state needs to be considered in any practical application. In other words, there is a trade-off between the maximum entanglement achieved and the time to get it, as exemplified by the numerical simulations in Fig. 3.

Quantum light emission.-So far, we have considered the two-mode entanglement in the coupled cavity system. The question now is how to extract this entanglement. In doing so, we study the architecture depicted in Fig. 1(b). For simplicity, we assumed that each resonator is locally coupled to the transmission line; see Fig. 1(c). In general, the coupling will be both through mutual inductance and capacitance. In the weak coupling limit, we end up with the usual RWA-type interaction $[43,44]$ : $H=i \sum_{k} \sqrt{\gamma N / 4 \pi^{2} v} \int d \omega\left[b^{\dagger}(\omega) a_{k}-\right.$ H.c. $]$, where $\gamma$ stands for the line-resonator coupling strength. For details, see the

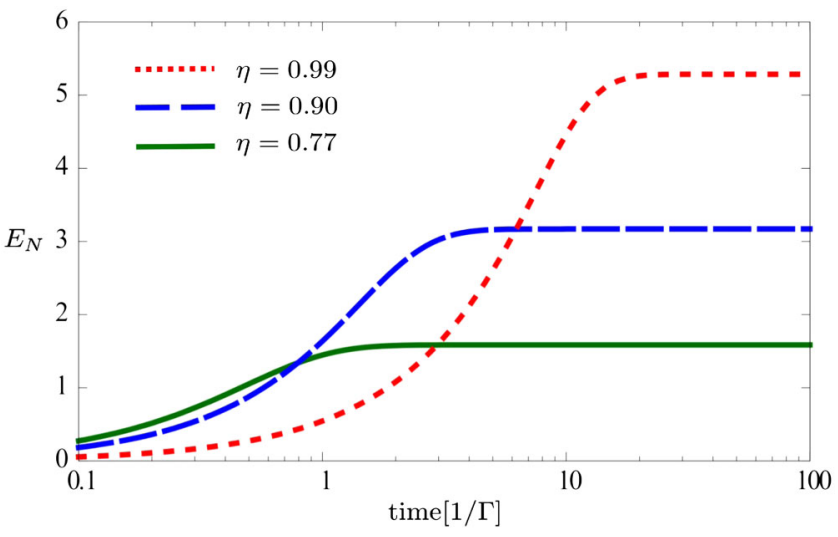

FIG. 3 (color online). Time evolution of the logarithmic negativity $E_{N}$ for $\mathcal{E}_{k,-k+q}=0$ and different values $\eta$.
Supplemental Material [38]. Now, using the input-output formalism, the fields in the transmission line before and after interacting with the array can be related through $[38,44]$

$$
b_{k}^{\text {in }}(t)=b_{k}^{\text {out }}(t)-\frac{1}{d} \sqrt{\frac{\gamma v}{N}} e^{-i(k v / d) t} a_{k},
$$

where $b_{k}^{\mathrm{in}}(t)=1 / 2 \pi \int_{(k-\delta) v / d}^{(k+\delta) v / d} d \omega e^{-i \omega t} b_{0}(\omega), \delta=\pi / N$, $d$ are the resonator lengths, and $v=1 / \sqrt{l_{0} c_{0}}$ the transmission line velocity $\left(l_{0}, c_{0}\right.$ are the inductance and capacitance per unit of length in the transmission line). This is the final result of the Letter. By considering the extended coupling, the resonator field modes are mapped onto the line with a resolution in frequency $\sim 1 / N$ ( $N$ is the number of oscillators). Therefore, the system presented here works as a source of chiral quantum light.

Conclusions.-In this Letter, we have introduced a model presenting criticality driven by dissipation. The model is based in the driven JCL. Our proposal uses simple elements, such as qubits with a tunable gap [45,46], coupled resonators arrays [24,25], and external drivings, which are standard in state-of-the-art CQED experiments. We argue that it could be implemented thusly and tested using recent advances in the field of microwave state tomography $[11,47,48]$. Some numbers for the qubit and resonator parameters already reported in the literature that could be used to test our results may be: $\epsilon / \omega_{r}=10$, $g / \omega_{r} \cong 10^{-2}, \Gamma / \omega_{r} \cong 1, \Delta / \omega_{r} \cong 10^{-2}$, and $J / \Delta \cong 1$ [38]. Furthermore, as detailed in the Supplemental Material [38], we have tested the feasibility of our proposal by (i) testing the approximations done to obtain the effective master equation (6) and (ii) checking the robustness of the state against the spread in the experimental parameters. We have done numerical investigations and, within a $10 \%$ error in the qubit gap, the amount of entanglement matches that obtained without errors in Eq. (10).

The potential interest of our work goes beyond the field of many body physics driven by dissipation. With the discussed feasibility and robustness, the state generated has a practical application. We have described how to use this nontrivial stationary state as a bright source of entangled light. This source features interesting properties: it is directional, with entangled modes that can be spatially separated; it is broadband, entangling different modes and frequencies, and the only parameters that need to be tuned are the intensity and the frequency of the external driving. Our ideas may also be useful in a variety of schemes recently introduced in the broader field of many-body quantum optical systems; see Ref. [49] (atomic ensembles), Ref. [50] (trapped ions), and Ref. [51] (quantum dots coupled to plasmonic cavities).

We acknowledge support from the Spanish DGICYT under Projects No. FIS2009-10061 and No. FIS2011-25167, by the Aragón (Grupo FENOL), QUITEMAD S2009-ESP-1594, and the EU Project PROMISCE. D. P. is supported by RyC Contract No. Y200200074. 
[1] S. L. Braunstein and P. van Loock, Rev. Mod. Phys. 77, 513 (2005).

[2] C. M. Caves, K. S. Thorne, R.W.P. Drever, V. D. Sandberg, and M. Zimmermann, Rev. Mod. Phys. 52, 341 (1980).

[3] D. F. Walls and G. J. Milburn, Quantum Optics (Springer, New York, 1994).

[4] A. Zagoskin, E. Ilichev, M. McCutcheon, J. Young, and F. Nori, Phys. Rev. Lett. 101, 253602 (2008).

[5] M. A. Castellanos-Beltran, K. D. Irwin, G. C. Hilton, L. R. Vale, and K. W. Lehnert, Nat. Phys. 4, 929 (2008).

[6] C. Eichler, D. Bozyigit, C. Lang, M. Baur, L. Steffen, J. M. Fink, S. Filipp, and A. Wallraff, Phys. Rev. Lett. 107, 113601 (2011).

[7] N. Bergeal, F. Schackert, L. Frunzio, and M. H. Devoret, Phys. Rev. Lett. 108, 123902 (2012).

[8] C. M. Wilson, G. Johansson, A. Pourkabirian, M. Simoen, J. R. Johansson, T. Duty, F. Nori, and P. Delsing, Nature (London) 479, 376 (2011).

[9] M. Hofheinz, H. Wang, M. Ansmann, R. C. Bialczak, E. Lucero, M. Neeley, A. D. O'Connell, D. Sank, J. Wenner, J. M. Martinis, and A. N. Cleland, Nature (London) 459, 546 (2009).

[10] E. Flurin, N. Roch, F. Mallet, M. Devoret, and B. Huard, Phys. Rev. Lett. 109, 183901 (2012).

[11] C. Eichler, D. Bozyigit, C. Lang, L. Steffen, J. Fink, and A. Wallraff, Phys. Rev. Lett. 106, 220503 (2011).

[12] K. W. Murch, U. Vool, D. Zhou, S. J. Weber, S. M. Girvin, and I. Siddiqi, Phys. Rev. Lett. 109, 183602 (2012).

[13] D. Marcos, A. Tomadin, S. Diehl, and P. Rabl, New J. Phys. 14, 055005 (2012).

[14] S. Diehl, A. Micheli, A. Kantian, B. Kraus, H. P. Büchler, and P. Zoller, Nat. Phys. 4, 878 (2008).

[15] B. Kraus, H. Büchler, S. Diehl, A. Kantian, A. Micheli, and P. Zoller, Phys. Rev. A 78, 042307 (2008).

[16] J. Eisert and T. Prosen, arXiv:1012.5013.

[17] A. Rakhmanov, A. Zagoskin, S. Savelev, and F. Nori, Phys. Rev. B 77, 144507 (2008).

[18] M. J. Hartmann, F. G. S. L. Brandão, and M. B. Plenio, Nat. Phys. 2, 849 (2006).

[19] A.D. Greentree, C. Tahan, J.H. Cole, and L.C.L. Hollenberg, Nat. Phys. 2, 856 (2006).

[20] D. Angelakis, M. Santos, and S. Bose, Phys. Rev. A 76, 031805 (2007).

[21] J. Koch and K. Le Hur, Phys. Rev. A 80 (2009)

[22] M. Leib and M. J. Hartmann, New J. Phys. 12, 093031 (2010).

[23] T. Hümmer, G. Reuther, P. Hänggi, and D. Zueco, Phys. Rev. A 85, 052320 (2012).

[24] D. Underwood, W. Shanks, J. Koch, and A. Houck, Phys. Rev. A 86, 023837 (2012).

[25] A. A. Houck, H. E. Türeci, and J. Koch, Nat. Phys. 8, 292 (2012).

[26] For all purposes, we can focus on the case of zero temperature.

[27] J. I. Cirac, R. Blatt, P. Zoller, and W. D. Phillips, Phys. Rev. A 46, 2668 (1992).

[28] J. I. Cirac, A. S. Parkins, R. Blatt, and P. Zoller, Phys. Rev. Lett. 70, 556 (1993).
[29] C.C. Gerry and E.E. Hach, Phys. Lett. A 174, 185 (1993).

[30] L. Gilles, B. M. Garraway, and P. L. Knight, Phys. Rev. A 49, 2785 (1994).

[31] N. Syassen, D. M. Bauer, M. Lettner, T. Volz, D. Dietze, J. J. García-Ripoll, J. I. Cirac, G. Rempe, and S. Dürr, Science 320, 1329 (2008).

[32] M. B. Plenio, S. F. Huelga, A. Beige, and P. L. Knight, Phys. Rev. A 59, 2468 (1999).

[33] M. B. Plenio and S. F. Huelga, Phys. Rev. Lett. 88, 197901 (2002).

[34] H. Krauter, C. Muschik, K. Jensen, W. Wasilewski, J. Petersen, J. Cirac, and E. Polzik, Phys. Rev. Lett. 107, 080503 (2011).

[35] C. Muschik, E. Polzik, and J. Cirac, Phys. Rev. A 83, 052312 (2011).

[36] A. Rivas and S.F. Huelga, Open Quantum Systems: An Introduction, Springer Briefs in Physics (Springer, Berlin, 2011), p. 100.

[37] F. Verstraete, M. M. Wolf, and J. Ignacio Cirac, Nat. Phys. 5, 633 (2009).

[38] See Supplemental Material at http://link.aps.org/ supplemental/10.1103/PhysRevLett.111.073602 for details on the derivation of the master equation and its validity, for the entanglement calculation, the input-ouput, and for the numerical computations including the spreading in the parameters.

[39] D. Porras and J. García-Ripoll, Phys. Rev. Lett. 108, 043602 (2012).

[40] $e^{i z \sin \theta}=\sum_{n=-\infty}^{\infty} J_{n}(z) e^{i n \theta}$ with $J_{n}(z)$ the Bessel function of first class.

[41] A. Einstein, B. Podolsky, and N. Rosen, Phys. Rev. 47, 777 (1935).

[42] E. M. Kessler, G. Giedke, A. Imamoglu, S. F. Yelin, M. D. Lukin, and J. I. Cirac, Phys. Rev. A 86, 012116 (2012).

[43] B. Peropadre, D. Zueco, F. Wulschner, F. Deppe, A. Marx, R. Gross, and J. J. García-Ripolll, Phys. Rev. B 87, 134504 (2013).

[44] C. W. Gardiner and M. J. Collett, Phys. Rev. A 31, 3761 (1985).

[45] F. G. Paauw, A. Fedorov, C. J. P. M Harmans, and J. Mooij, Phys. Rev. Lett. 102, 090501 (2009).

[46] M. J. Schwarz, J. Goetz, Z. Jiang, T. Niemczyk, F. Deppe, A. Marx, and R. Gross, New J. Phys. 15, 045001 (2013).

[47] D. Bozyigit, C. Lang, L. Steffen, J. M. Fink, C. Eichler, M. Baur, R. Bianchetti, P. J. Leek, S. Filipp, M. P. da Silva, A. Blais, and A. Wallraff, Nat. Phys. 7, 154 (2010).

[48] E. P. Menzel, F. Deppe, M. Mariantoni, M. A. Araque Caballero, A. Baust, T. Niemczyk, E. Hoffmann, A. Marx, E. Solano, and R. Gross, Phys. Rev. Lett. 105, 100401 (2010).

[49] D. E. Chang, L. Jiang, A. V. Gorshkov, and H. J. Kimble, New J. Phys. 14, 063003 (2012).

[50] A. Bermudez, T. Schaetz, and M. B. Plenio, Phys. Rev. Lett. 110, 110502 (2013).

[51] A. González-Tudela and D. Porras, Phys. Rev. Lett. 110, 080502 (2013). 